\title{
Analysis of systems with slope restricted nonlinearities using externally positive Zames-Falb multipliers
}

\author{
Matthew C. Turner, Member, IEEE, Ross Drummond
}

\begin{abstract}
This paper proposes an approach for assessing the stability of feedback interconnections where one element is a static slope-restricted nonlinearity and the other element is a linear system. The approach is based on the use of ZamesFalb multipliers where the dynamic portion of the multiplier is chosen as an externally positive non-causal transfer function. By restricting attention to a sub-set of these multipliers, a set of pure LMI conditions is obtained which requires no initial paramterisation by the user. A useful by-product of using externally positive systems is that the results are applicable to non-odd slope restricted nonlinearities, which is not the case for all classes of Zames-Falb multipliers.
\end{abstract}

\section{INTRODUCTION}

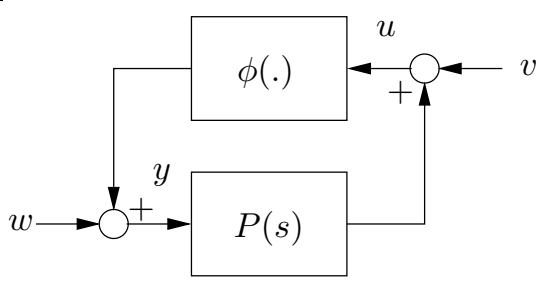

Fig. 1. System under consideration

This paper considers the stability of the interconnection depicted in Figure 1 where $P(s) \in \mathcal{R} \mathcal{H}_{\infty}$ is a single-inputsingle-output (SISO) linear time invariant system and $\phi($.$) is$ a static, time-invariant, slope-restricted nonlinearity. Various approaches have been proposed for the analysis of Figure 1. These tend to be either Lyapunov-based (recent references are [19], [12], [26], [31] - see also the book [14]) or inputoutput stability based, where the Circle and Popov Criteria are popular. Perhaps the most general method for studying the stability of the system in Figure 1 is using so-called Zames-Falb multipliers which were introduced by [18] (see [6] for a perspective on this), with the precise technical conditions finalised shortly afterwards [33]. After years in the research wilderness, researchers, emboldened by the greater computational facilities of today, have recently begun to look at Zames-Falb multipliers again ([9], [1], [10]).

The main difficulty in the analysis of systems using ZamesFalb multipliers is the troubling mixture of time and frequency domain conditions which arise: the multipliers must be chosen such that a passivity (actually positivity) condition is satisfied,

M.C. Turner is with the Department of Engineering, University of Leicester, Leicester, LE1 7RH, UK. +44 116252 2548. Email mct6@le.ac.uk

R. Drummond is with the Department of Engineering Science, University of Oxford, OX1 3PJ, UK. Email ross.drummondleng.ox.ac.uk whilst also meeting a time domain $\mathcal{L}_{1}$ bound on their impulse response. It appears to be challenging to satisfy these conditions simultaneously, non-conservatively and efficiently. Some interesting examples, inspired by [18], are discussed in [6]

Several searches for Zames-Falb multipliers satisfying (simultaneously) positivity and $\mathcal{L}_{1}$ bounds have been proposed and these depend on the parametrisation of the multiplier used. The first and most popular of these was proposed by [8], where the multiplier was chosen to have a transfer function consisting of a sum of first order terms; the user was required to choose pole locations and order. This approach has been advocated and refined [32], but as illustrated in [6] there are some instances for which the approach is inherently unsuitable, and in some cases analysis using this approach can be unsatisfactory.

An alternative parametrisation of Zames-Falb multipliers was proposed in [28] and developed in [5], [27]. This approach fixed the order of the multiplier to that of the plant, and used a bound on the $\mathcal{L}_{1}$ norm proposed in [24]. The resulting procedure required no user-defined choices but did require a line search over a scalar parameter; in addition, the $\mathcal{L}_{1}$ norm bound used was well-known to be conservative. A further down-side to this approach was that the analysis was only valid for odd nonlinearities.

Another approach is that of [11] where a search over nonrational multipliers was proposed. This approach was further developed in [7]. As shown in [5], [6], this approach can be competitive, but sometimes it is conservative and numerically unreliable. The extensions of the Zames-Falb analysis tools to multivariable systems have been discussed in [23], [15] and tools for the search over the class of MIMO ZamesFalb multipliers have been proposed in [9], [29], [10]. It is probably fair to say that a complete solution to the problem originally considered by O'Shea some fifty years ago - that of finding a multiplier rendering a system with a slope restricted nonlinearity stable - is not yet available.

This paper uses the parametrisation of the multiplier suggested in [28], [5] but enforces the $\mathcal{L}_{1}$ bound in a more convenient manner. The work takes advantage of recent results on positive systems, and in particular external positivity. This allows one to replace the line search which appears in the algorithms of [28], [5] by a linear matrix inequality (LMI). Consequently the arising stability analysis algorithm is completely convex, leading in many cases to significantly shorter computational times compared with other Zames-Falb based approaches. In addition, in some examples the results offered by the approach proposed here are competitive with the state-of-the-art. 


\section{A. Notation}

Notation is standard. For a square matrix $M$ the notation $M>$ $0(\geq 0)$ means the matrix is symmetric and positive (semi-) definite. Negative definiteness is defined similarly. $I$ denotes the identity matrix, of appropriate dimensions; $I_{m}$ denotes the identity matrix of size $m$.

This paper mainly considers scalar-valued signals. The convolution of two scalar signals $x(t)$ and $y(t)$ is denoted $x(t) \star y(t)$. For scalar signals, $x(t) \in \mathbb{R}$, the $\mathcal{L}_{1}$ norm is defined as

$$
\|x\|_{1}:=\int_{-\infty}^{\infty}|x(t)| d t
$$

With some abuse of notation, $\|H(s)\|_{1}$ also denotes the $\mathcal{L}_{1}$ norm of the impulse response associated with the transfer function $H(s)$. A signal $x(t) \in \mathcal{L}_{1}$ if $\|x\|_{1}$ is finite.

The $\mathcal{L}_{2}$ norm of a signal $x(t) \in \mathbb{R}$ is defined as

$$
\|x\|_{2}:=\int_{-\infty}^{\infty}|x(t)|^{2} d t
$$

$x(t) \in \mathcal{L}_{2}$ if $\|x\|_{2}$ is finite. Signals $x(t)$ which are zero for all $t<0$ belong to the subspace $\mathcal{L}_{2}[0, \infty)$.

For scalar signals $x(t) \in \mathbb{R}$, the $\mathcal{L}_{\infty}$ norm is defined as

$$
\|x\|_{\infty}:=\sup _{t \in(-\infty, \infty)}|x(t)|
$$

The space of real rational transfer functions bounded on the imaginary axis is denoted by $\mathcal{R} \mathcal{L}_{\infty}$, with norm defined as

$$
\|G\|_{\mathcal{L}_{\infty}}=\sup _{\omega \in(-\infty, \infty)}|G(j \omega)|
$$

The subspace $\mathcal{R H}_{\infty}\left(\mathcal{R} \mathcal{H}_{\infty}^{\perp}\right) \subset \mathcal{R} \mathcal{L}_{\infty}$ denotes those transfer functions analytic in the open right (left) half plane.

A system $G(s)$ with impulse response $g(t)$ is causal (anticausal) if $g(t)=0$ for all $t<0(t>0)$. A system $G(s)$ is said to be bounded if its impulse response, $g(t) \in \mathcal{L}_{1}$. If $G(s) \in \mathcal{R} \mathcal{H}_{\infty}$ it is interpreted as a bounded causal operator; $G(s) \in \mathcal{R H}_{\infty}^{\perp}$ is a bounded anti-causal operator. $G^{\sim}(s)=$ $G(-s)$ is the adjoint of the transfer function $G(s) ; G(s) \in$ $\mathcal{R H}_{\infty}$ implies $G(-s) \in \mathcal{R} \mathcal{H}_{\infty}^{\perp}$.

\section{Stability Analysis Using ZAMES-FALB MULTIPLIERS}

Consider Figure 1 in which $P(s)$ is a finite dimensional linear time invariant (FDLTI) system with state-space realisation

$$
P(s) \sim\left[\begin{array}{c|c}
A_{p} & B_{p} \\
\hline C_{p} & D_{p}
\end{array}\right]
$$

where $A_{p} \in \mathbb{R}^{n_{p} \times n_{p}}, B_{p} \in \mathbb{R}^{n_{p} \times 1}, C_{p} \in \mathbb{R}^{1 \times n_{p}}, D_{p} \in \mathbb{R}$.

The nonlinear element $\phi():. \mathbb{R} \mapsto \mathbb{R}$ is assumed to be slope restricted; its slope (generalised derivative) is assumed to be confined to a certain interval, that is

$$
0 \leq \frac{\phi(x)-\phi(y)}{x-y} \leq \alpha \quad \forall x, y \in \mathbb{R}, \quad \alpha>0
$$

For brevity, the notation $\partial \phi \in[0, \alpha]$ means that the nonlinearity satisfies inequality (6). It is assumed that appropriate loop-shifting has been carried out on the system such that the lower bound on the slope is zero. Formally, the nonlinearity belongs to one of the classes of nonlinearity defined below.

Definition 1: $\phi():. \mathbb{R} \mapsto \mathbb{R}$ is said to belong to $\mathcal{N}_{[0, \alpha]}^{S}$ if

i) It is bounded.

ii) It has slope restriction $\partial \phi \in[0, \alpha], \alpha>0$.

$\phi($.$) is said to belong to \mathcal{N}_{[0, \alpha]}^{S, \text { odd }}$ if, in addition, it is odd.

Unlike in some foregoing work ([28]), $\phi($.$) may not be odd.$ The slope bound, $\partial \phi \in[0, \alpha]$ implies the sector bound, $\phi \in$ Sector $[0, \alpha]$ if $\phi(0)=0$ (e.g. [27]), meaning Circle and Popov multipliers may be included in the analysis of such systems.

Zames-Falb multipliers are used to establish stability conditions for the system in Figure 1. A convenient way of viewing the main results of [33] is through the use of integralquadratic-constraints (IQC's) [16]. This enables Zames-Falb multipliers to be augmented with other stability multipliers, such as those of the Popov and Circle Criteria, and also enables one to cast the search for non-causal multipliers in the same framework as a purely causal search [25], [20]. This is not obvious from the original results of [33] and will be exploited in this paper.

It is well-known ([16]) that if $\phi \in \mathcal{N}_{[0, \alpha]}^{S}$, it satisfies the IQC

$$
\int_{-\infty}^{\infty}\left[\begin{array}{c}
\hat{u}(j \omega) \\
\hat{\phi(u)}(j \omega)
\end{array}\right]^{\star} \Pi(j \omega)\left[\begin{array}{c}
\hat{u}(j \omega) \\
\hat{\phi(u)}(j \omega)
\end{array}\right] d \omega \geq 0
$$

where $\hat{u}(j \omega)$ and $\widehat{\phi(u)}(j \omega)$ are the Fourier Transforms of $u(t)$ and $\phi(u(t))$ respectively, and $\Pi(s)=\Pi_{Z F}(s)$ is given by

$$
\Pi_{Z F}(s)=\left[\begin{array}{cc}
0 & \alpha M^{\sim}(s) \\
\alpha M(s) & -M^{\sim}(s)-M(s)
\end{array}\right]
$$

The transfer function $M(s)$ is the so-called "Zames-Falb multiplier" ([33]). The class of Zames-Falb multipliers is not restricted to rational transfer functions in general (see [33]) but for this paper the rational subset of these, $\mathcal{M}_{R}$ is sufficient.

Definition 2: A transfer function $M(s):=H_{0}-H(s) \in \mathcal{R} \mathcal{L}_{\infty}$ is said to belong to the set $\mathcal{M}_{R}^{\text {odd }}$ if $H_{0}>0$ and $H(s) \in \mathcal{L}_{1}$ is such that $\|H(s)\|_{1} \leq H_{0}$. If, in addition, the impulse response of $H(s), h(t) \geq 0$ for all $t \in(-\infty, \infty), M(s)$ is said to belong to the set $\mathcal{M}_{R}$.

When $M(s) \in \mathcal{M}_{R}$, the IQC (7)-(8) captures the largest class of rational multipliers for $\phi(.) \in \mathcal{N}_{[0, \alpha]}^{S}$. For the case when $\phi($. is vector valued, the reader is referred to [9], [15]. The class of $\mathcal{M}_{R}$ is large and includes multipliers $M(s)$ of arbitrarily high order. In practice, lower order multipliers are used.

\section{A. Stability and IQC factorisation}

The main stability result of Zames and Falb [33] is stated in the IQC framework for the reasons set out earlier. Generally, Zames-Falb multipliers may include both causal and anticausal terms. However, in the IQC framework, it is possible to factorise the IQC so that all anti-causal terms appear together [25]. Then, because the main IQC stability condition is evaluated on the imaginary axis, it effectively allows the anti-causal terms to be handled using the causal approach of 
[28], [30]. In this paper "stability of Figure 1" means that, for all exogenous signals $v, w \in \mathcal{L}_{2}[0, \infty)$, the loop signals $u, y \in \mathcal{L}_{2}[0, \infty)$. Under mild conditions, this is equivalent to asymptotic stability in the state-space context.

Theorem 1 (Adapted from [16]): Consider Figure 1 where $P(s) \in \mathcal{R H}_{\infty}$ and $\phi \in \mathcal{N}_{[0, \alpha]}^{S}$ satisfies the IQC defined by (7) and (8) where $M(s) \in \mathcal{M}_{R}$. Assume that the closed loop system is well-posed. Then the system is stable if there exists an $\epsilon>0$ such that

$$
\left[\begin{array}{c}
P(j \omega) \\
I
\end{array}\right]^{\star} \Pi_{Z F}(j \omega)\left[\begin{array}{c}
P(j \omega) \\
I
\end{array}\right]<-\epsilon I \quad \forall \omega \in \mathbb{R}
$$

Thus stability of the system essentially reduces to finding suitable $M(s) \in \mathcal{M}_{R}$ such that inequality (9) holds. This means: (i) in order to test (9) one has to search for an appropriate, and potentially high-order, $M(s) \in \mathcal{M}_{R}$, which may be computationally challenging; and (ii) finding a suitable $M(s) \in \mathcal{M}_{R}$ involves a combination of frequency-domain (9) and time domain $\left(\|H\|_{1} \leq H_{0}\right.$ and $\left.h(t) \geq 0\right)$ conditions. The initial approach to this [8] was to choose a basis of $H(s)$ for which the $\mathcal{L}_{1}$ bound was known and then to calculate the associated gains to ensure that $\|H\|_{1} \leq H_{0}$ and (9) is satisfied. For low-order choices of $M(s)$ this can be useful but the approach is essentially ad hoc. A more recent approach has been to choose $M(s)$ to be either causal [28], or anti-causal [5], but plant order. Then, via a change of variables, a suitable $M(s)$ can be chosen using LMI's and a line search.

Although the plant-order searches advocated in [28], [27], [5] are entirely systematic, all suffer from conservatism: the search is conducted over either causal or anti-causal multipliers. These searches can be useful, and sometimes produce tight results, but their restriction to causal or anti-causal multipliers is limiting. Noting the observations of [25] this problem can be surmounted by using an alternative factorisation of the multiplier. Consider again the multiplier $\Pi(s)=\Pi_{Z F}(s)$ :

$$
\Pi_{Z F}(s)=\left[\begin{array}{cc}
0 & \alpha M \sim(s) \\
\alpha M(s) & -M^{\sim}(s)-M(s)
\end{array}\right]
$$

Let $M(s) \in \mathcal{M}_{R}$ have the following structure

$$
M(s)=H_{0}-H_{c}(s)-H_{a}^{\sim}(s), \quad H_{c}(s), H_{a}(s) \in \mathcal{R H}_{\infty}
$$

In the above equation, $H_{c}(s) \in \mathcal{R} \mathcal{H}_{\infty}$ represents the causal part of the multiplier and $H_{a}^{\sim}(s) \in \mathcal{R} \mathcal{H}_{\infty}^{\perp}$ its anti-causal part. Grouping causal and anti-causal terms, it follows that

$$
\Pi_{Z F}(s)=Y(s)+Y^{\sim}(s)
$$

where $Y(s) \in \mathcal{R} \mathcal{H}_{\infty}$ is defined as

$$
Y(s):=\left[\begin{array}{cc}
0 & -\alpha H_{a}(s) \\
\alpha\left(H_{0}-H_{c}(s)\right) & -\left(H_{0}-H_{a}(s)-H_{c}(s)\right)
\end{array}\right]
$$

Setting $\Psi(s)=\left[\begin{array}{ll}Y(s) & I\end{array}\right]^{\prime} \in \mathcal{R} \mathcal{H}_{\infty}$ it then follows that

$$
\Pi_{Z F}(s)=\Psi^{\sim}(s) W \Psi(s) \quad W=\left[\begin{array}{cc}
0 & I_{2} \\
I_{2} & 0
\end{array}\right]
$$

With this factorisation of $\Pi_{Z F}(s)$, Theorem 1 can then be re-written as Corollary 1 below.
Corollary 1: Consider Figure 1 where $P(s) \in \mathcal{R H}_{\infty}$ and $\phi \in \mathcal{N}_{[0, \alpha]}^{S}$ satisfies the IQC defined by (7) and (14) where $M(s) \in \mathcal{M}_{R}$ is given by (11). Assume that the closed loop system is well-posed. Then the system is stable if there exists an $\epsilon>0$ such that

$$
L(j \omega)^{*} W L(j \omega)<-\epsilon I \quad \forall \omega \in \mathbb{R}
$$

where

$$
L(s)=\left[\begin{array}{c}
-\alpha H_{a}(s) \\
\left(H_{0}-H_{c}(s)(\alpha P(s)-1)+H_{a}(s)\right. \\
P(s) \\
1
\end{array}\right]
$$

Proof: The factorisation (14) in Theorem 1 provides the result after some algebra. Note that $L(s)$ contains no anti-causal terms.

\section{B. Externally positive systems}

This subsection introduces several facts about positive systems which will be used subsequently. Positive systems have gained popularity because some problems which are intractable for arbitrary linear systems become tractable for positive systems ([22]). Throughout this section we consider a FDLTI system, $G(s)$, with state-space representation

$$
G(s) \sim\left[\begin{array}{l|l}
A & B \\
\hline C & D
\end{array}\right]
$$

It is assumed that $G(s)$ is SISO, with $A \in \mathbb{R}^{n \times n}, B, C^{\prime} \in \mathbb{R}^{n}$ and $D \in \mathbb{R}$. An externally positive system is defined below.

Definition 3: [22] $G(s)$ is said to be externally positive if its impulse response $g(t) \geq 0 \quad \forall t \in(-\infty, \infty)$.

Fact 1: A causal linear system $G(s) \sim(A, B, C, D)$ is externally positive if its impulse response $g(t)$ is non-negative for all $t \in[0, \infty)$; that is

$$
g(t)=C e^{A t} B+D \delta(t) \geq 0 \quad \forall t \geq 0
$$

Determining whether a given linear system is externally positive is, generally, not trivial [17], [2]. Much of the literature on positive systems considers internally positive systems which means that for any non-negative initial condition, the state remains non-negative thereafter. This requires $A$ to be a Metzler matrix (i.e. the off-diagonal elements are non-negative) and all elements of $B$ to be non-negative. If, in addition, all elements of $C$ and $D$ are non-negative, this is sufficient for $G(s) \sim(A, B, C, D)$ to be externally positive. Unfortunately, internal positivity is a fragile property which is generally not preserved by a state-similarity transformation. Therefore, we note the following fact which ensures external positivity.

Fact 2: Assume $G(s) \in \mathcal{R H}_{\infty}$ with state-space realisation $(A, B, C, D)$. Let $A=A^{\prime}, B=C^{\prime}$ and $D \geq 0$, then $G(s)$ is an externally positive linear system. 
Proof: $G(s) \in \mathcal{R} \mathcal{H}_{\infty}$ only has a non-zero impulse response on $t \in[0, \infty)$ i.e.

$$
\begin{aligned}
g(t)=C e^{A t} B+D \delta(t) & =C e^{A t / 2} e^{A t / 2} C^{\prime}+D \delta(t) \\
& =C e^{A t / 2} e^{A^{\prime} t / 2} C^{\prime}+D \delta(t) \\
& =\left\|C e^{A t / 2}\right\|^{2}+D \delta(t)
\end{aligned}
$$

One of the key advantages of positive systems is the fact that their norms are bounded by their D.C. gains. The following result for causal positive systems is essentially a re-statement of part of Proposition 3 from [22].

Lemma 1: Let $G(s) \in \mathcal{R} \mathcal{H}_{\infty}$, and have state-space realisation $(A, B, C, 0)$. If $G(s)$ is externally positive then:

$$
\|G(s)\|_{1}=-C A^{-1} B=G(0)
$$

Proof: The proof is repeated for convenience. Note that the $\mathcal{L}_{\infty}$ gain of $G(s)$ is equivalent to the $\mathcal{L}_{1}$ norm of its impulse response, $g(t)$. Taking $y(t)=g(t) \star w(t)$, we have

$$
\begin{aligned}
\|y(t)\|_{\infty} & =\sup _{t}\left|\int_{0}^{\infty} g(\tau) w(t-\tau) d \tau\right| \\
& \leq \sup _{t} \int_{0}^{\infty}|g(\tau) \| w(t-\tau)| d \tau \\
& \leq \int_{0}^{\infty}|g(\tau)| d \tau \quad\|w\|_{\infty} \\
& =\int_{0}^{\infty} g(\tau) d \tau \quad\|w\|_{\infty} \\
& =\left[C A^{-1} e^{A t} B\right]_{0}^{\infty} \quad\|w\|_{\infty}
\end{aligned}
$$

Now, because $G(s) \in \mathcal{R H}_{\infty}$, then $A$ is Hurwitz so

$$
\left[C A^{-1} e^{A t} B\right]_{0}^{\infty}=-C A^{-1} B=G(0) \geq\|G(s)\|_{1}
$$

However, there exists a $w(t)$ (a constant) such that $\|y(t)\|_{\infty}=$ $G(0)\|w(t)\|_{\infty}$, hence equality holds: $G(0)=\|G(s)\|_{1}$

A parallel lemma can be derived for the case where $G(s)$ is anti-causal, i.e. $G(s) \in \mathcal{R H}_{\infty}^{\perp}$.

Lemma 2: Let $G(s) \in \mathcal{R} \mathcal{H}_{\infty}^{\perp}$, and have state-space realisation $(A, B, C, 0)$. If $G(s)$ is externally positive then:

$$
\|G(s)\|_{1}=-C A^{-1} B=G(0)
$$

Proof: Let $G^{\sim}(s)=G(-s)$ be the adjoint of $G(s)$. Then $G(s) \in \mathcal{R H}_{\infty}^{\perp}$ implies $G^{\sim}(s) \in \mathcal{R H}_{\infty}$. As $\|G(s)\|_{1}=$ $\left\|G^{\sim}(s)\right\|_{1}$, it remains to apply Lemma 1 to $\left\|G^{\sim}(s)\right\|_{1}$. Note that

$$
G^{\sim}(s)=-C(s I+A)^{-1} B
$$

so $G^{\sim}(0)=-C A^{-1} B=G(0)$, which proves the lemma

Zames-Falb multipliers are, in general non-causal. The following lemma shows how the $\mathcal{L}_{1}$ norm of an externally positive but non-causal system can be bounded by the $\mathcal{L}_{1}$ norm of an externally positive causal system. This lemma will be used in establishing the main results of the paper.
Lemma 3: Let $H(s)=H_{c}(s)+H_{a}^{\sim}(s)$ be a non-causal transfer function where $H_{c}(s), H_{a}(s) \in \mathcal{R} \mathcal{H}_{\infty}$ and where $H_{c}(s)$ and $H_{a}^{\sim}(s)$ are both externally positive. Then $\bar{H}(s):=H_{c}(s)+$ $H_{a}(s)$ is also externally positive and, furthermore,

$$
\|H(s)\|_{1} \leq\|\bar{H}(s)\|_{1}
$$

Proof: If $H_{a}^{\sim}(s)$ is externally positive, this implies that $h_{a}^{\sim}(t) \geq 0 \quad \forall t \in(-\infty, 0]$, which implies that $h_{a}(t) \geq 0 \quad \forall t \in$ $[0, \infty)$. Hence $H_{a}(s)$ is externally positive. Together with external positivity of $H_{c}(s)$, this implies $\bar{H}(s)$ is externally positive. To see the $\mathcal{L}_{1}$ bound note that

$$
\begin{aligned}
\|H(s)\|_{1} & =\left\|H_{c}(s)+H_{a}^{\sim}(s)\right\|_{1} \\
& \leq\left\|H_{c}(s)\right\|_{1}+\left\|H_{a}^{\sim}(s)\right\|_{1} \\
& =H_{c}(0)+H_{a}^{\sim}(0) \\
& =H_{c}(0)+H_{a}(0) \\
& =\left\|H_{c}(s)+H_{a}(s)\right\|_{1}=\|\bar{H}(s)\|_{1}
\end{aligned}
$$

where the penultimate equality follows because $\bar{H}(s) \in \mathcal{R} \mathcal{H}_{\infty}$ and is externally positive.

\section{MAin Results}

\section{A. Stability using external positivity}

The aim of this section is to translate the frequency inequality in Corollary 1 to an LMI; and to replace the $\mathcal{L}_{1}$ condition appearing in the same corollary with another LMI. The main result is preceded by an intermediate result for clarity.

Proposition 1: Assume, $P(s) \in \mathcal{R H}_{\infty}, \phi(.) \in \mathcal{N}_{[0, \alpha]}^{S}$ and that the multiplier, $M(s)$ is structured as described in equation (11) with $H_{c}, H_{a} \in \mathcal{R} \mathcal{H}_{\infty}$. Assume the following state-space realisations:

$$
\begin{aligned}
& H_{c}(s) \sim\left[\begin{array}{c|c}
A_{c} & B_{c} \\
\hline C_{c} & 0
\end{array}\right] \\
& H_{a}(s) \sim\left[\begin{array}{c|c}
A_{a} & B_{a} \\
\hline C_{a} & 0
\end{array}\right]
\end{aligned}
$$

Assume further that $\left\|H_{c}+H_{a}^{\sim}\right\|_{1}<H_{0}$ and the impulse response of $H(s)=H_{c}(s)+H_{a}^{\sim}(s)$ is positive for all $t \in(-\infty, \infty)$. Then if there exists a real symmetric matrix $P=P^{\prime}$ satisfying the following matrix inequality

$$
\left[\begin{array}{cc}
\tilde{A}^{\prime} P+P \tilde{A}+\tilde{C}^{\prime} W \tilde{C} & P \tilde{B}+\tilde{C}^{\prime} W \tilde{D} \\
\star & \tilde{D}^{\prime} W \tilde{D}
\end{array}\right]<0
$$

where $W$ is given in (14) and $\tilde{A}, \tilde{B}, \tilde{C}$ and $\tilde{D}$ are given by

$$
\left[\begin{array}{c|c}
\tilde{A} & \tilde{B} \\
\hline \tilde{C} & \tilde{D}
\end{array}\right]=\left[\begin{array}{ccc|c}
A_{p} & 0 & 0 & B_{p} \\
\alpha B_{p} C_{p} & A_{c} & 0 & -B_{c} \\
0 & 0 & A_{a} & B_{a} \\
\hline 0 & 0 & -\alpha C_{a} & 0 \\
\alpha H_{0} C_{p} & -C_{c} & C_{a} & H_{0}\left(\alpha D_{p}-1\right) \\
C_{p} & 0 & 0 & D_{p} \\
0 & 0 & 0 & 1
\end{array}\right]
$$

the system in Figure 1 is stable.

Proof: The proof is essentially an application of the KYP Lemma [21] to Corollary 1. Noting $M(s)=H_{0}-H_{c}(s)-$ 
$H_{a}^{\sim}(s)$, it follows that $L(s)$ (given in equation (16) of Corollary 1) has a state-space realisation $L(s) \sim(\tilde{A}, \tilde{B}, \tilde{C}, \tilde{D})$ where the state-space matrices are defined in equation (36).

Applying the KYP Lemma to inequality (15) in Corollary 1 results in the (nonlinear) matrix inequality (35). Stability follows by noting the assumptions $\left\|H_{c}(s)+H_{a}^{\sim}(s)\right\|_{1} \leq H_{0}$ and $h(t) \geq 0 \quad \forall t \in(-\infty, \infty)$ imply $M(s)$ belongs to $\mathcal{M}_{R}$ and thus the conditions of Corollary 1 are satisfied.

Remark - properness. As noted in [4], without loss of generality, $H_{c}(s)$ and $H_{a}(s)$ are assumed strictly proper.

The next step is to re-write both the matrix inequality (35) and the $\mathcal{L}_{1}$ condition on the multiplier as LMI's. This is achieved by using a high-order Zames-Falb multiplier approach [30], and also the positive systems theory of Section II-B.

Proposition 2: Assume $P(s) \in \mathcal{R} \mathcal{H}_{\infty}$ and that $\phi(.) \in \mathcal{N}_{[0, \alpha]}^{\mathrm{S}}$. Then the system in Figure 1 is stable if there exist positive definite matrices $\mathbf{S}_{\mathbf{1 1}}, \mathbf{P}_{\mathbf{1 1}}$ and $\mathbf{N}, \mathbf{A}_{c}$ and $\mathbf{A}_{a}$, a positive scalar $\mathbf{H}_{0}$ and unstructured matrices $\mathbf{C}_{\mathbf{c}}$ and $\mathbf{C}_{\mathbf{a}}$ such that inequalities (37), (39) and (40) below hold.

$$
\begin{gathered}
{\left[\begin{array}{ccc}
\mathbf{H}_{0} & \mathbf{C}_{\mathbf{c}}{ }^{\prime} & \mathbf{C}_{\mathbf{a}}{ }^{\prime} \\
\star & \mathbf{A}_{c} & 0 \\
\star & 0 & \mathbf{A}_{a}
\end{array}\right] \geq 0} \\
\mathbf{P}_{11}-\mathbf{S}_{11}-\mathbf{N}>0
\end{gathered}
$$

Proof of Proposition 2: The proof of this proposition, similar to [28], [5], has two main parts: the translation of the matrix inequality (35) into (37); and the translation of the requirement that $M(s) \in \mathcal{M}_{R}$ to inequality (39), plus a final part to verify that an assumption of positive definiteness indeed holds. The derivation of the matrix inequalities requires an assumption on the structure of the multiplier. From Proposition 1, the multiplier is assumed to have the structure of $M(s)$ given in equation (11) with causal part $H_{c}(s) \in \mathcal{R} \mathcal{H}_{\infty}$ and anti-causal part $H_{a}^{\sim}(s) \in \mathcal{R} \mathcal{H}_{\infty}^{\perp}$. It is also assumed that both the causal and anti-causal parts have order equal to that of the plant, viz $\operatorname{ord}\left[H_{c}(s)\right]=\operatorname{ord}\left[H_{a}(s)\right]=n_{p}$.

Part 1: Main inequality. Consider inequality (35) where the state-space matrices $(\tilde{A}, \tilde{B}, \tilde{C}, \tilde{D})$ are given in equation (36). Due to the structure of the multiplier described above, it follows that the symmetric matrix $P \in \mathbb{R}^{3 n_{p} \times 3 n_{p}}$. Under the additional assumption that $P>0$, let $P$ and $Q:=P^{-1}$ be partitioned into $n_{p} \times n_{p}$ sub-matrices:

$\left[\begin{array}{lll}Q_{11} & Q_{12} & Q_{13} \\ Q_{12}^{\prime} & Q_{22} & Q_{23} \\ Q_{13}^{\prime} & Q_{23}^{\prime} & Q_{33}\end{array}\right]\left[\begin{array}{ccc}\mathbf{P}_{11} & P_{12} & P_{13} \\ P_{12}^{\prime} & P_{22} & 0 \\ P_{13}^{\prime} & 0 & P_{33}\end{array}\right]=\left[\begin{array}{ccc}I & 0 & 0 \\ 0 & I & 0 \\ 0 & 0 & I\end{array}\right]$

where, $P_{12}, P_{13}, Q_{12}$ and $Q_{13}$ are full rank. Later it will be verified that the inequalities in the theorem do indeed ensure that $P>0$.This particular structure of $P$ is assumed to enable convex conditions to be obtained; its limitation will be discussed subsequently. Thus, introducing the matrices

$$
\Pi_{1}:=\left[\begin{array}{ccc}
Q_{11} & I & 0 \\
Q_{12}^{\prime} & 0 & 0 \\
Q_{13}^{\prime} & 0 & I
\end{array}\right] \quad \Pi_{2}:=\left[\begin{array}{ccc}
I & 0 & 0 \\
\mathbf{P}_{11} & P_{12} & P_{13} \\
P_{13}^{\prime} & 0 & P_{33}
\end{array}\right]
$$

it follows that $\Pi_{1}^{\prime} P=\Pi_{2}$. This can be considered to be an extension of Scherer's change of variables introduced in [24].

Applying, the congruence transformation $\operatorname{diag}\left(\Pi_{1}^{\prime}, I\right)$ to inequality (35) gives the following equivalent inequality

$$
\left[\begin{array}{cc}
\Pi_{1}^{\prime} \tilde{A}^{\prime} \Pi_{2}^{\prime}+\Pi_{2} \tilde{A} \Pi_{1}+\Pi_{1}^{\prime} \tilde{C}^{\prime} W \tilde{C} \Pi_{1} & \Pi_{2} \tilde{B}+\Pi_{1} \tilde{C}^{\prime} \\
\star & \tilde{D}^{\prime} W \tilde{D}
\end{array}\right]<0
$$

Applying a further congruence transformation

$$
\operatorname{diag}\left(\mathbf{S}_{11}, I, I, I\right) \quad \mathbf{S}_{11}:=Q_{11}^{-1}
$$

to inequality (43) yields, after some algebra [28], inequality (38) where the following matrix variables have been defined:

$$
\begin{aligned}
\mathbf{A}_{\mathbf{c}} & :=P_{12} A_{c} Q_{12}^{\prime} \mathbf{S}_{\mathbf{1 1}} \\
\mathbf{B}_{\mathbf{c}} & :=P_{12} B_{c} \\
\mathbf{C}_{\mathbf{c}} & :=C_{c} Q_{12}^{\prime} \mathbf{S}_{\mathbf{1 1}} \\
\mathbf{A}_{\mathbf{a}} & :=-P_{13} A_{a} P_{33}^{-1} P_{13}^{\prime} \\
\mathbf{B}_{\mathbf{a}} & :=-P_{13} B_{a} \\
\mathbf{C}_{\mathbf{a}} & :=C_{a} P_{33}^{-1} P_{13}^{\prime} \\
\mathbf{N} & :=P_{13} P_{33}^{-1} P_{13}^{\prime}
\end{aligned}
$$

In the above derivation, it is useful to note, from identity (41), that $Q_{13}=-Q_{11} P_{13} P_{33}^{-1}$. Setting $\mathbf{B}_{\mathbf{c}}=-\mathbf{C}_{\mathbf{c}}{ }^{\prime}, \mathbf{B}_{\mathbf{a}}=-\mathbf{C}_{\mathbf{a}}{ }^{\prime}$, $\mathbf{A}_{\mathbf{c}}=\mathbf{A}_{\mathbf{c}}{ }^{\prime}$ and $\mathbf{A}_{\mathbf{a}}=\mathbf{A}_{\mathbf{a}}{ }^{\prime}$ then yields inequality (37) in the proposition; this choice will be justified in part 2 of the proof.

Part 2: The $\mathcal{L}_{1}$ condition. This part of the proof gives an $\mathcal{L}_{1}$ bound on the norm of $H(s)$, thereby ensuring that it belongs to $\mathcal{M}_{R}$. This LMI is derived under the assumption that $H(s)$ is externally positive; it is then proved for the choice made that this is indeed the case.

It is convenient to work with the transfer function $\bar{H}(s)=$ $H_{c}(s)+H_{a}(s)$, where $H_{c}, H_{a} \in \mathcal{R} \mathcal{H}_{\infty}$. Using equations (45) - (50), a state-space realisation of $\bar{H}(s)$ is given as

$$
\bar{H}(s) \sim\left[\begin{array}{c|c}
A_{\bar{H}} & B_{\bar{H}} \\
\hline C_{\bar{H}} & 0
\end{array}\right]=\left[\begin{array}{c|c}
U \mathbf{A}_{\bar{H}} V & U \mathbf{B}_{\bar{H}} \\
\hline \mathbf{C}_{\bar{H}} V & 0
\end{array}\right]
$$

where

$$
\begin{aligned}
{\left[\begin{array}{c|c}
\mathbf{A}_{\bar{H}} & \mathbf{B}_{\bar{H}} \\
\hline \mathbf{C}_{\bar{H}} & 0
\end{array}\right]=\left[\begin{array}{cc|c}
\mathbf{A}_{\mathbf{c}} & 0 & \mathbf{B}_{\mathbf{c}} \\
0 & \mathbf{A}_{\mathbf{a}} & \mathbf{B}_{\mathbf{a}} \\
\hline \mathbf{C}_{\mathbf{c}} & \mathbf{C}_{\mathbf{a}} & 0
\end{array}\right] } \\
U=\left[\begin{array}{cc}
P_{12}^{-1} & 0 \\
0 & -P_{13}^{-1}
\end{array}\right] V=\left[\begin{array}{cc}
\left(Q_{12}^{\prime} S_{11}\right)^{-1} & 0 \\
0 & \left(P_{33}^{-1} P_{13}^{\prime}\right)^{-1}
\end{array}\right]
\end{aligned}
$$

Using the similarity transformation

$$
T=\left[\begin{array}{cc}
-P_{12}^{\prime} & 0 \\
0 & P_{13}^{-1} \mathbf{N}
\end{array}\right]^{-1}
$$

an equivalent realisation of $H(s)$ is then

$$
\bar{H}(s) \sim\left[\begin{array}{c|c}
T U \mathbf{A}_{\bar{H}} V T^{-1} & T U \mathbf{B}_{\bar{H}} \\
\hline \mathbf{C}_{\bar{H}} V T^{-1} & 0
\end{array}\right]
$$

Using the identity (41), the following expressions arise:

$$
\begin{aligned}
& 0=Q_{11} P_{12}+Q_{12} P_{22} \\
& 0=Q_{11} P_{13}+Q_{13} P_{33} \\
& I=Q_{11} \mathbf{P}_{11}+Q_{12} P_{12}^{\prime}+Q_{13} P_{13}^{\prime}
\end{aligned}
$$




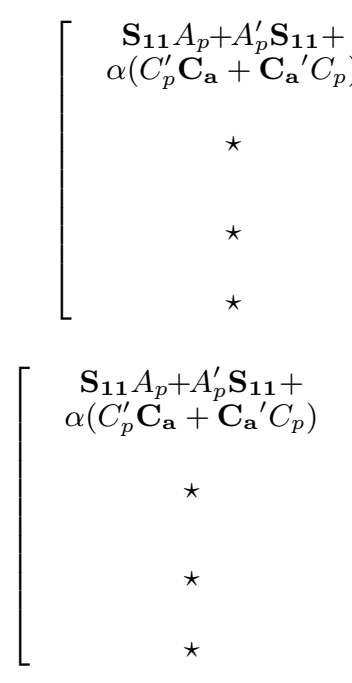

Setting $P_{22}=I$ and recalling that $\mathbf{S}_{11}=Q_{11}^{-1}$ then yields

$$
\begin{aligned}
Q_{12} & =-Q_{11} P_{12} \\
S_{11} Q_{13} & =-P_{13} P_{33}^{-1} \\
P_{12} P_{12}^{\prime} & =\mathbf{P}_{11}-\mathbf{S}_{11}-\mathbf{N}
\end{aligned}
$$

Using these equations, it then follows that

$$
\bar{H}(s) \sim\left[\begin{array}{c|c}
-X \mathbf{A}_{\bar{H}} & -X \mathbf{B}_{\bar{H}} \\
\hline \mathbf{C}_{\bar{H}} & 0
\end{array}\right]
$$

where

$$
X:=\left[\begin{array}{cc}
\left(\mathbf{P}_{11}-\mathbf{S}_{11}-\mathbf{N}\right) & 0 \\
0 & \mathbf{N}
\end{array}\right]
$$

Temporarily assume that $\bar{H}(s)$ is externally positive. Then, because $\bar{H}(s)$ is causal, it follows from Lemma 1, and the realisation (63) that $\|\bar{H}(s)\|_{1} \leq H_{0}$ if

$$
\begin{aligned}
& -\mathbf{C}_{\bar{H}} \mathbf{A}_{\bar{H}}^{-1} X^{-1} X \mathbf{B}_{\bar{H}} \leq H_{0} \\
& \Leftrightarrow H_{0}+\mathbf{C}_{\bar{H}} \mathbf{A}_{\bar{H}}^{-1} \mathbf{B}_{\bar{H}} \geq 0
\end{aligned}
$$

Now, setting $\mathbf{A}_{\bar{H}}=\mathbf{A}_{\bar{H}}^{\prime}$ and $\mathbf{B}_{\bar{H}}=-\mathbf{C}_{\bar{H}}$ ( implies $\mathbf{B}_{\mathbf{c}}=-\mathbf{C}_{\mathbf{c}}{ }^{\prime}, \mathbf{B}_{\mathbf{a}}=-\mathbf{C}_{\mathbf{a}}{ }^{\prime}, \mathbf{A}_{\mathbf{c}}=\mathbf{A}_{\mathbf{c}}{ }^{\prime}$ and $\mathbf{A}_{\mathbf{a}}=\mathbf{A}_{\mathbf{a}}{ }^{\prime}$ as mentioned in Part 1) gives

$$
H_{0}-\mathbf{C}_{\bar{H}} \mathbf{A}_{\bar{H}}^{-1} \mathbf{C}_{\bar{H}}=\underline{\underline{\underline{\underline{\rho}}}}
$$

This inequality implies that $\|\bar{H}(s)\|_{1} \leq H_{0}$ and thus, by Lemma 3 , if $H(s)$ is also externally positive, $\|H(s)\|_{1}<H_{0}$. Inequality (67) then gives LMI (39) in the proposition.

The temporary assumption of external positivity of $\bar{H}(s)$ and $H(s)$ is now removed. LMI (40) in the proposition implies that $X$ is positive definite and hence that $X^{\frac{1}{2}}$ exists. Thus using $X^{\frac{1}{2}}$ as a similarity transformation, an equivalent state-space realisation of $\bar{H}(s)$ is

$$
\bar{H}(s) \sim\left[\begin{array}{c|c}
\hat{A}_{\bar{H}} & \hat{B}_{\bar{H}} \\
\hline \hat{C}_{\bar{H}} & 0
\end{array}\right]=\left[\begin{array}{c|c}
-X^{\frac{1}{2}} \mathbf{A}_{\bar{H}} X^{\frac{1}{2}} & -X^{\frac{1}{2}} \mathbf{B}_{\bar{H}} \\
\hline \mathbf{C}_{\bar{H}} X^{\frac{1}{2}} & 0
\end{array}\right]
$$

Therefore, with the choices $\mathbf{B}_{\bar{H}}=-\stackrel{-\underline{\underline{E}}}{\underline{E}}$ and $\mathbf{A}_{\bar{H}}=\mathbf{A}_{\bar{H}}^{\prime}$, it is clear that $\hat{A}_{\bar{H}}=\hat{A}_{\bar{H}}^{\prime}$ and $\hat{B}_{\bar{H}}=\mathrm{C}_{\bar{H}}^{\prime}$; by Fact $2, \bar{H}(s)$ is

$$
\left.\begin{array}{cc}
A_{p}^{\prime} \mathbf{N}+\mathbf{A}_{\mathbf{a}}{ }^{\prime} & \mathbf{S}_{\mathbf{1 1}} B_{p}-\mathbf{C}_{\mathbf{c}}{ }^{\prime}+\alpha C_{p}^{\prime} \mathbf{H}_{0} \\
-\alpha C_{p}^{\prime} \mathbf{C}_{\mathbf{a}} & +\mathbf{C}_{\mathbf{a}}{ }^{\prime}\left(\alpha D_{p}-1\right) \\
A_{p}^{\prime} \mathbf{N}-\mathbf{A}_{\mathbf{a}} & \mathbf{P}_{\mathbf{1 1}} B_{p}+\mathbf{C}_{\mathbf{a}}{ }^{\prime}+\mathbf{C}_{\mathbf{c}}{ }^{\prime} \\
-\alpha C_{p}^{\prime} \mathbf{C}_{\mathbf{a}} & +\alpha C_{p}^{\prime} \mathbf{H}_{0} \\
-2 \mathbf{A}_{\mathbf{a}} & \mathbf{N} B_{p}+\mathbf{C}_{\mathbf{a}}{ }^{\prime} \\
\star & -\mathbf{C}_{\mathbf{a}}{ }^{\prime}\left(\alpha D_{p}-1\right) \\
\star & 2 \mathbf{H}_{0}\left(\alpha D_{p}-1\right)
\end{array}\right]<0
$$

indeed externally positive. Finally because $\bar{H}(s)=H_{c}(s)+$ $H_{a}(s)$ where $H_{c}(s), H_{a}(s) \in \mathcal{R} \mathcal{H}_{\infty}$, are externally positive, it follows that $H(s)=H_{c}(s)+H_{a}^{\sim}(s)$ is also externally positive.

Part 3: Nonsingularity of $P$

In Part 1 of the proof it was assumed that $P>0$. Here, we show that satisfaction of the LMI's in the proposition guarantees that this is indeed the case. $P>0$ is equivalent to $\Pi_{1}^{\prime} P \Pi_{1}=\Pi_{2} \Pi_{1}>0$, which can be written as

$$
\Pi_{2} \Pi_{1}=\left[\begin{array}{ccc}
Q_{11} & I & 0 \\
I & \mathbf{P}_{11} & P_{13} \\
0 & P_{13}^{\prime} & P_{33}
\end{array}\right]>0
$$

By the Schur Complement this is equivalent to

$$
\left[\begin{array}{cc}
Q_{11} & I \\
I & \mathbf{P}_{11}-P_{13} P_{33}^{-1} P_{13}^{\prime}
\end{array}\right]>0
$$

Applying the Schur Complement again and noting equation (51) this is equivalent to inequality (40) as required.

Remark - tractability. Proposition 2 gives entirely convex conditions for stability analysis using Zames-Falb multipliers and requires no line search (which appears in the conditions of [28], [5]) and no choice of multiplier pole locations and order (which is required in the conditions of [8], [10]).

Remark - conservatism. There are several sources of conservatism present in Proposition 2. The first is the constraint on the multiplier: $H(s)=H_{c}(s)+H_{a}^{\sim}(s)$ is constrained to be externally positive. This is not only a fundamental constraint on the multiplier, but also its enforcement using Fact 2 requires the matrices $\mathbf{A}_{c}$ and $\mathbf{A}_{\mathbf{a}}$ to be symmetric, and that $\mathbf{B}_{\mathbf{c}}=-\mathbf{C}_{\mathbf{c}}{ }^{\prime}$ and $\mathbf{B}_{\mathbf{a}}=-\mathbf{C}_{\mathbf{a}}{ }^{\prime}$; these are sufficient but not necessary conditions for $H(s)$ to be externally positive. Conservatism also arises from the structure of the matrix $P$ because $P_{23}$ is assumed zero. This structure is assumed because it allows Proposition 2 to be expressed entirely in convex conditions: if $P_{23} \neq 0$, these conditions become nonconvex. The size of of $P \in \mathbb{R}^{3 n_{p} \times 3 n_{p}}$ is also specific and again is assumed to enable convex and elegant conditions to be obtained. Of course, both of these structural conditions could be dropped, but this would result in considerable complication of Proposition 2: conservatism is traded for tractability. 
Remark - multiplier. $\bar{H}(s)$ can be constructed as $\bar{H}(s)=$ $H_{c}(s)+H_{a}(s)$ using equation (68). As both $\mathbf{A}_{\mathbf{a}}$ and $\mathbf{A}_{\mathbf{c}}$ are positive definite, the realisation (68) implies $H_{c}(s), H_{a}(s) \in$ $\mathcal{R H}_{\infty}$ as expected. Therefore $H_{a}^{\sim}(s)=H_{a}(-s) \in \mathcal{R H}_{\infty}^{\perp}$, again as expected, which allows the construction of the multiplier $M(s)=H_{0}-H_{c}(s)-H_{a}^{\sim}(s)$.

\section{B. Including Popov Multipliers}

Frequently the nonlinearity $\phi($.$) may satisfy an additional$ sector condition; that is

$$
\phi \in \operatorname{Sector}[0, \beta]
$$

This is certainly the case when $\phi(0)=0$ because the sloperestriction then directly implies a sector bound: $\phi \in \mathcal{N}_{[0, \alpha]}^{S} \Rightarrow$ $\phi \in \operatorname{Sector}[0, \alpha]$. More generally a tighter bound on the sector might be available: $\beta<\alpha$. In either case, it may be possible to reduce the conservatism of the stability analysis by augmenting the Zames-Falb multiplier with a Popov multiplier [27]. In the IQC framework,the Popov multiplier is given by

$$
\Pi_{P}(s)=\left[\begin{array}{cc}
0 & (\beta \nu+\eta s)^{\sim} \\
(\beta \nu+\eta s) & -2 \nu
\end{array}\right]
$$

Thus, with a Popov multiplier, $\phi($.$) satisfies the IQC given in$ equation (7) but with $\Pi(s)$ given by [13], [27]:

$$
\begin{aligned}
\Pi(s)= & \Pi_{Z F}(s)+\Pi_{P}(s) \\
= & {\left[\begin{array}{cc}
0 & (\nu \beta+\eta s+\alpha M(s))^{\sim} \\
(\nu \beta+\eta s+\alpha M(s)) & -2 \nu-M^{\sim}(s)-M(s)
\end{array}\right], } \\
& \nu \in \mathbb{R}_{+}, \quad \eta \in \mathbb{R}, \quad M(s) \in \mathcal{M}_{R}
\end{aligned}
$$

Following a similar procedure to that described in Section III-A then yields the following result.

Proposition 3: Assume $P(s) \in \mathcal{R} \mathcal{H}_{\infty}$ with $D_{p}=0$ and that $\phi(.) \in \mathcal{N}_{[0, \alpha]}^{\mathrm{S}}$ and $\phi \in \operatorname{Sector}[0, \beta]$. Then the system in Figure 1 is stable if there exist positive definite matrices $\mathbf{S}_{\mathbf{1 1}}, \mathbf{P}_{\mathbf{1 1}}$, $\mathbf{N}, \mathbf{A}_{\mathbf{c}}, \mathbf{A}_{\mathbf{a}}$, positive scalars $\mathbf{H}_{0}$ and $\nu$, unstructured matrices $\mathbf{C}_{\mathbf{c}}$ and $\mathbf{C}_{\mathbf{a}}$, and an indefinite scalar $\eta$, such that inequalities (39) - (40) from Prop. 2 hold and in addition inequality (75) overleaf also holds.

Proof: The proof is omitted but follows along the same lines as described in Section III-A. The noteworthy features of the proof are the addition of the Popov terms in the fourth row/column in inequality (75), which are incorporated in a similar manner as described in [27].

Remark - improvements. The Popov multiplier was added to the Zames-Falb multiplier in [27] in order to improve the effectiveness of the search. As noted in [3], [6] the addition of the Popov multiplier in an otherwise causal search (see [27]) can be viewed as an extra anti-causal element; the addition of the Popov multiplier in an otherwise anti-causal search (see [5]) can be viewed as an extra causal element. However, in the non-causal search proposed here, it may be anticipated that the Popov terms may not yield improvements because the search, by its nature, features both causal and anti-causal elements; this will be verified later.

\section{NUMERICAL EXAMPLES}

Despite some complacency in the literature, determining which approach to Zames-Falb multiplier computation is "best" is not trivial [6]. Perhaps the most widely used search is that of [8] and indeed this is useful for many examples. However, often its success depends on the use of a high-order multiplier which may create numerical problems and limits its use in practice.

The examples used here are, as in [6], inspired by those suggested by [18]. They show that in some cases the search over externally positive Zames-Falb multipliers can be both attractive and competitive. The plants considered have the form

$$
P_{i}(s)=\frac{1}{\left(s^{2}+2 \zeta_{i} \omega_{n} s+\omega_{n}^{2}\right)^{2}}
$$

with $\omega_{n}=10$ and $\zeta_{i} \in\{0.05,0.1,0.15\}$. For simplicity the sector and slope bounds are assumed identical: $\alpha=\beta$.

Table I shows the results obtained. Comparisons are made with the work of [19], the causal/anti-causal searches of [28], [5] and also those of [8]. Popov multipliers have been included in the causal/anti-causal searches of [27], [5] and [8]. For the results of [8], an 18th order Zames-Falb was used with half the poles located at $s=-1$ and half at $s=1$, as in [6]. The set-up used was an Intel i7 920 PC equipped with 6GB RAM, running Linux Mint 13, Matlab 15a and using the LMI solver from the Robust Control Toolbox. It is interesting to note that

- For these examples the bounds on $\alpha$ offered by Propositions 2 and 3 are less conservative than the results given by [8] with a Popov multiplier.

- The results of [28], [27], [5] offer superior results to Propositions 2 and 3 for $\zeta=0.05$ and $\zeta=0.1$, but are valid only when the nonlinearity $\phi($.$) is odd.$

- Propositions 2 and 3 offer the least conservative results (narrowly) for the case when $\zeta=0.15$.

- As mentioned earlier, because the search proposed in Proposition 2 contains causal and anti-causal terms, one would not expect a search which, in addition, features a Popov multiplier (Proposition 3 ) to provide any improvements [3]. This is indeed the case, with Popov multipliers not offering any improvement, at least for the case when the slope and sector bound are identical i.e. $\alpha=\beta$.

- The square brackets in Table I show the computational time required to compute the largest slope size $(\alpha)$ for each method. It can be seen that the calculations involving the positive Zames-Falb multipliers require the least computation time in all cases, except for the results obtained from Park's approach [19]. It is anticipated that this trend would be more evident for higher-order systems.

\section{CONClusion}

The properties of externally positive linear systems have been exploited in Zames-Falb multiplier analysis. The main appeal of the work is that the search over this class of multipliers is purely convex, with no additional choices required from the user. The analysis is valid, by the nature of the multipliers, for non-odd nonlinearities. In some instances, the results obtained using this approach are competitive with the state-of-the-art. 


$$
\left[\begin{array}{cccc}
\mathbf{S}_{\mathbf{1 1}} A_{p}+A_{p}^{\prime} \mathbf{S}_{\mathbf{1 1}}+ & \mathbf{S}_{\mathbf{1 1}} A_{p}+A_{p}^{\prime} \mathbf{P}_{\mathbf{1 1}}+\mathbf{A}_{\mathbf{c}}{ }^{\prime}+\mathbf{A}_{\mathbf{a}}{ }^{\prime} & A_{p}^{\prime} \mathbf{N}+\mathbf{A}_{\mathbf{a}}{ }^{\prime} & \mathbf{S}_{\mathbf{1 1}} B_{p}-\mathbf{C}_{\mathbf{c}}{ }^{\prime}-\mathbf{C}_{\mathbf{a}}{ }^{\prime} \\
\alpha\left(C_{p}^{\prime} \mathbf{C}_{\mathbf{a}}+\mathbf{C}_{\mathbf{a}}{ }^{\prime} C_{p}\right) & -\alpha\left(C_{p}^{\prime} \mathbf{C}_{\mathbf{c}}-\mathbf{C}_{\mathbf{a}}{ }^{\prime} C_{p}\right) & -\alpha C_{p}^{\prime} \mathbf{C}_{\mathbf{a}} & +\alpha \mathbf{H}_{0} C_{p}^{\prime}+\beta \nu C_{p}^{\prime}+\eta A_{p}^{\prime} C_{p}^{\prime} \\
\star & A_{p}^{\prime} \mathbf{P}_{\mathbf{1 1}}+\mathbf{P}_{\mathbf{1 1}} A_{p} & A_{p}^{\prime} \mathbf{N}-\mathbf{A}_{\mathbf{a}} & \mathbf{P}_{\mathbf{1 1}} B_{p}+\mathbf{C}_{\mathbf{c}}{ }^{\prime}+\mathbf{C}_{\mathbf{a}}{ }^{\prime} \\
\star & -\alpha\left(\mathbf{C}_{\mathbf{c}}{ }^{\prime} C_{p}+C_{p}^{\prime} \mathbf{C}_{\mathbf{c}}\right) & -\alpha C_{p}^{\prime} \mathbf{C}_{\mathbf{a}} & +\alpha \mathbf{H}_{0} C_{p}^{\prime}+\beta \nu C_{p}^{\prime}+\eta A_{p}^{\prime} C_{p}^{\prime} \\
\star & \star & -2 \mathbf{A}_{\mathbf{a}} & \mathbf{N} B_{p}+2 \mathbf{C}_{\mathbf{a}}{ }^{\prime} \\
\star & \star & \star & -2\left(\mathbf{H}_{0}+\nu\right)+\eta\left(C_{p} B_{p}+B_{p}^{\prime} C_{p}^{\prime}\right)
\end{array}\right]<0
$$

TABLE I

MAXIMUM SLOPE SIZES, $\alpha$, FOR WHICH STABILITY IS GUARANTEED. THE SQUARE BRACKETED FIGURE INDICATES THE NUMBER OF SECONDS COMPUTATION TIME REQUIRED.

\begin{tabular}{|c|c|c|c|c|}
\hline \multirow[t]{2}{*}{ Method } & \multicolumn{3}{|c|}{ Example } & Class of $\phi()$. \\
\hline & $\zeta=0.05$ & $\zeta=0.10$ & $\zeta=0.15$ & \\
\hline Park [19] & $799.9995[0.50]$ & $3199.9981[0.59]$ & $7199.9767[0.40]$ & $\mathcal{N}_{[0}^{S}$ \\
\hline Z-F (causal plant order) [28] & $1819.9832[53.19]$ & $4972.3331[51.51]$ & $7495.5867[50.51]$ & $\mathcal{N}_{[0, \alpha]}^{S, o d d}$ \\
\hline Z-F (causal plant order) + Popov [27] & 1944.9471 [287.09] & $\mathbf{5 7 2 1 . 7 9 4 8}[263.05]$ & $8679.4280[275.70]$ & $\mathcal{N}_{\text {[0, }}^{S,}$ \\
\hline Z-F (anti-causal plant order) [5] & $1819.9832[53.67]$ & $4972.3331[51.70]$ & $7495.5867[50.63]$ & $\mathcal{N}_{[0, \alpha]}^{S, \text { odd }}$ \\
\hline Z-F (anti-causal plant order) + Popov [5] & $1944.9471[286.47]$ & $\mathbf{5 7 2 1 . 7 9 4 8}[263.30]$ & $8679.4280[275.62]$ & $\mathcal{N}_{[0, \alpha]}^{S, o d d}$ \\
\hline Z-F (non-causal, positive) Prop.2 & $920.8806[8.79]$ & $4091.6504[5.75]$ & $8736.7144[4.75]$ & $\mathcal{N}_{[0}^{S}$ \\
\hline Z-F (non-causal, positive) + Popov Prop. 3 & $920.8806[22.78]$ & $4091.6504[22.42]$ & $\mathbf{8 7 3 6 . 7 1 4 4}[14.97]$ & $\mathcal{N}_{[0, \alpha]}^{S}$ \\
\hline Z-F Chen and Wen $[8]+$ Popov & $871.2747[67.04]$ & $3620.8743[64.32]$ & $8043.6727[64.72]$ & $\mathcal{N}_{[0, \alpha]}^{S}$ \\
\hline
\end{tabular}

\section{ACKNOWLEDGEMENTS}

The authors thank Dr. Peter Seiler from the University of Minnesota for comments on an early version of this paper.

\section{REFERENCES}

[1] D.A. Altshuller. Delay-integral-quadratic constraints and stability multipliers for systems with MIMO nonlinearities. IEEE Trans. Aut. Control, 56(4):738-747, 2011.

[2] L. Benvenuti and L. Farina. A tutorial on the positive realization problem. IEEE Trans. Aut. Control, 49(5):651-664, 2004.

[3] J. Carrasco, W.P. Heath, and A. Lanzon. Equivalence between classes of multipliers for slope-restricted nonlinearities. Automatica, 2012.

[4] J. Carrasco, W.P. Heath, G. Li, and A. Lanzon. Comments on 'On the existence of stable, causal multipliers for systems with slope-restricted nonlinearities'. IEEE Trans. Aut. Control, 2012.

[5] J. Carrasco, M. Maya-Gonzalez, A. Lanzon, and W.P. Heath. LMI searches for anticausal and noncausal rational Zames-Falb multipliers. Systems \& Control Letters, 70:17-22, 2014.

[6] J. Carrasco, M.C. Turner, and W.P. Heath. Zames-Falb multipliers for absolute stability: From O'Shea's contribution to convex searches. European Journal of Control, 28:1-19, 2016.

[7] M. Chang, R. Mancera, and M.G. Safonov. Computation of Zames-Falb multipliers revisited. IEEE Trans. Aut. Control, 57(4):1024-1029, 2012.

[8] X. Chen. and T. Wen. Robustness analysis of LTI systems with structured incrementally sector bounded nonlinearities. Proc. American Control Conf., pages 3883-3887, 1995.

[9] F.J. D'Amato, M.A. Rotea, A.V. Megretski, and U.T. Jonsson. New results for analysis of systems with repeated nonlinearities. Automatica, 37(6):739-747, 2001.

[10] M. Fetzer and C.W. Scherer. Full-block multipliers for repeated, slope restricted scalar nonlinearities. Int. J. Robust Nonlininear Control, 2017.

[11] P.B. Gapski and J.C. Geromel. A convex approach to the absolute stability problem. IEEE Trans. Aut. Control, 39(9):1929-1932, 1994.

[12] W.P. Heath and G. Li. Lyapunov functions for the multivariable Popov criterion with indefinite multipliers. Automatica, 45(12):2977-2981, 2009.

[13] U. Jonsson. Stability analysis with Popov multipliers and integral quadratic constraints. Systems and Control Letters, 31:85-92, 1997.

[14] G.A. Leonov, D.V. Ponomarenko, and V.B. Smirnova. FrequencyDomain Methods for Nonlinear Analysis. World Scientific, Singapore, 1996.

[15] R. Mancera and M.G. Safonov. All repeated multipliers for MIMO nonlinearities. Systems and Control Letters, 54(4):389-397, 2005.
[16] A.V. Megretski and A. Rantzer. System analysis via integral quadratic constraints. IEEE Trans. Aut. Control, 42(6):819-830, 1997.

[17] Y. Ohta, H. Maeda, and S. Kodoma. Reachability, observability and realizability of continuous-time positive systems. SIAM J. Control and Optimization, 22(2):171-180, 1984.

[18] R. O'Shea. A combined frequency-time domain stability criterion for autonomous continuous systems. IEEE Trans. Aut. Control, 11(3):477484, 1966.

[19] P.G. Park. Stability criteria of sector and slope restricted Lur'e systems. IEEE Trans. Aut. Control, 47(2):308-313, 2002.

[20] H. Pfifer and P.J. Seiler. Robustness analysis of LPV systems using IQC's. In Proc. American Control Conf., Portland, 2014.

[21] A. Rantzer. On the Kalman-Yakubovich-Popov lemma. Systems and Control Letters, 28(1):7-10, 1996.

[22] A. Rantzer. Scalable control of positive systems. European Journal of Control, 24:72-80, 2015.

[23] M.G. Safonov and V.V. Kulkarni. Zames Falb multipliers for MIMO nonlinearities. Int. J. Robust and Nonlinear Control, 10(10):1025-1038, 2000.

[24] C. Scherer, P. Gahinet, and M. Chilali. Multiobjective output feedback control via LMI optimisation. IEEE Trans. Aut. Control, 42(7):896-911, 1997.

[25] P.J. Seiler. Stability analysis with dissipation inequalities and integral quadratic constraints. IEEE Trans. Aut. Control, 60(6):1704-1709, 2015.

[26] M.C. Turner and M. Kerr. Lyapunov functions and $\mathcal{L}_{2}$-gain bounds for systems with slope restricted nonlinearities. Systems \& Control Letters, 69:1-6, 2014

[27] M.C. Turner and M.L. Kerr. $\mathcal{L}_{2}$ gain bounds for systems with sector bounded and slope-restricted nonlinearities. Int. J. Robust and Nonlinear Control, 22(13):1505-1521, 2012.

[28] M.C. Turner, M.L. Kerr, and I. Postlethwaite. On the existence of stable, causal multipliers for systems with slope-restricted nonlinearities. IEEE Trans. Aut. Control, 54(11):2697-2702, 2009.

[29] M.C. Turner, M.L. Kerr, and J. Sofrony. Tractable stability analysis for systems containing repeated scalar slope-restricted nonlinearities. Int. J. Robust and Nonlinear Control, 25(7):971-986, 2015.

[30] M.C. Turner and J. Sofrony. High order Zames-Falb multiplier analysis. In Proc. European Control Conf., Zurich, 2013.

[31] G. Valmorbida, R. Drummond, and S.R. Duncan. Positivity conditions of Lyapunov functions for systems with slope restricted nonlinearities. In American Control Conference, pages 258-263, 2016.

[32] J. Veenman and C.W. Scherer. IQC-synthesis with general dynamic multipliers. Int. J. Robust and Nonlinear Control, 24(17):3027-3056, 2014.

[33] G. Zames and P.L. Falb. Stability conditions for systems with monotone and slope restricted nonlinearities. SIAM Journal of Control, 6(1):89108, 1968. 